\title{
Anti-Ro/SSA Antibodies May Be Responsible for Cerebellar Degeneration in Sjogren's Syndrome
}

\author{
Syuichi Tetsuka a, b, Tomohiro Suzuki ${ }^{\mathrm{a}}$, Tomoko Ogawa ${ }^{\mathrm{a}}$, \\ Ritsuo Hashimoto ${ }^{\text {a }}$, Hiroyuki Kato ${ }^{\mathrm{a}}$
}

\begin{abstract}
Background: Neurological disorders have been identified to be a common extraglandular manifestation of Sjogren's syndrome ( $\mathrm{SjS}$ ). Central nervous system (CNS) symptoms appear in about $5 \%$ of patients with $\mathrm{SjS}$. However, so far, only a few incidences of cerebellar degeneration have been reported, and the clinical features and pathological mechanisms associated with $\mathrm{SjS}$ remain to be unclear. Intramedullary production of anti-Ro/anti-SjS-related antigen A (SSA) has been observed in some patients with $\mathrm{SjS}$ patients who have CNS involvement, suggesting the involvement of anti- Ro/SSA antibodies as antineuronal antibodies in previous studies.
\end{abstract}

Methods: We recently treated cerebellar degeneration in a patient with $\mathrm{SjS}$. We analyzed the serum and cerebrospinal fluid (CSF) in order to detect anti-Ro/SSA and anti-La/anti-SjS-related antigen B (SSB) antibodies. We also searched the literature for previous case reports to evaluate the characteristics of cerebellar degeneration in patients with SjS. First, we have studied in mouse brain tissue and examined whether the Ro/SSA (Ro52/tripartite motif protein (TRIM)21) protein was expressed in the cerebellum of mice using immunohistochemistry.

Results: Although all patients that we found in the literature review and our patient 1 were positive for anti-Ro/SSA antibodies, some patients were also negative for anti-La/SSB antibodies. Anti-Ro/SSA antibodies were observed in both serum and CSF; however, anti-Ro/ SSA antibodies were negative in the CSF of patients with $\mathrm{SjS}$ without CNS involvement. Cerebellar atrophy was observed, and sequelae remained in the majority of patients. Autopsy findings indicated a selective loss of Purkinje cells. Ro52/TRIM21 expression was also detected throughout murine brains, including the hippocampus, cerebral cortex and cerebellum. High Ro52/TRIM21 expression was observed in the Purkinje cells.

Conclusions: We described the characteristics of cerebellar degen-

Manuscript submitted January 16, 2021, accepted January 26, 2021

Published online February 25, 2021

aDepartment of Neurology, International University of Health and Welfare Hospital, 537-3, Iguchi, Nasushiobara, Tochigi 329-2763, Japan

${ }^{b}$ Corresponding Author: Syuichi Tetsuka, Department of Neurology, International University of Health and Welfare Hospital, 537-3, Iguchi, Nasushiobara, Tochigi 329-2763, Japan. Email: syuichi@jichi.ac.jp

doi: https://doi.org/10.14740/jocmr4429 eration in patients with $\mathrm{SjS}$ and Ro52/TRIM21 expression in the Purkinje cells of murine cerebellar tissue sections. These outcomes indicate that anti-Ro/SSA antibodies were likely responsible for cerebellar degeneration in patients suffering from $\mathrm{SjS}$.

Keywords: Anti-Ro/SSA antibodies; Cerebellar degeneration; Purkinje cell; Ro52/TRIM21; Sjogren's syndrome

\section{Introduction}

Sjogren's syndrome ( $\mathrm{SjS}$ ) has been defined as an autoimmune disease in which the exocrine glands, primarily the salivary glands, are damaged. In addition, $\mathrm{SjS}$ is known to affect a wide variety of organs, including the skin, joints, nervous system, lungs, kidneys and digestive tract [1]. In particular, peripheral and central neurological symptoms can be evident in about $15 \%$ and $5 \%$ of patients with $\mathrm{SjS}$, respectively [2]. In the past decade, central nervous system (CNS) involvement in SjS has been observed more commonly than initially suspected, with disorders that include encephalitis, cognitive disorders, meningitis, myelitis and cerebellar degeneration. However, only a few reports of cerebellar degeneration have been described, and its clinical features and pathological mechanisms associated with $\mathrm{SjS}$ are yet to be determined.

Anti-Ro/anti-SjS-related antigen A (SSA) and anti-La/ anti-SjS-related antigen B (SSB) antibodies have been identified to be essential for the classification of $\mathrm{SjS}$ during diagnostic workups [3]. On the basis of molecular weights, Ro/SSA and $\mathrm{La} / \mathrm{SSB}$ antibodies target three cellular proteins, namely, Ro52 (also referred to as tripartite motif protein (TRIM)21), Ro60 and La48 [3]. Intramedullary production of anti-Ro52/ TRIM21 antibodies has been observed in some patients with SjS who have CNS involvement, suggesting the involvement of anti-Ro52/TRIM21 antibodies as antineuronal antibodies, and it has been reported that cerebrospinal fluid (CSF) anti-Ro/ SSA antibodies can serve as a biomarker for SjS-related CNS involvement [4]. However, an understanding on the molecular and pathological mechanisms behind autoantibodies in CNS manifestations of $\mathrm{SjS}$, including cerebellar degeneration, remains to be lacking; thus, further investigations are required to clarify their associations.

We recently treated cerebellar degeneration in a patient with SjS. We analyzed serum and CSF to determine any pres- 
ence of anti-Ro/SSA and anti-La/SSB antibodies. We also performed a literature review to assess the clinical characteristics, diagnostic methods and therapeutic strategies used for patients with SjS who have cerebellar degeneration. Moreover, we examined the expression of autoantigens (potential autoantibody target sites) in the murine cerebellar tissue sections to elucidate the molecular and pathological mechanisms of cerebellar degeneration in these patients.

\section{Materials and Methods}

\section{Patients}

Written informed consent was obtained from the patients in these case presentations, including for the accompanying images in the figures.

\section{Patient 1 (SjS with cerebellar degeneration)}

A 36-year-old male patient with progressive gait imbalance for 2 weeks was admitted to our neurology department. He had no family history of gait disturbance and neurological disorders and no history of exposure to toxins or drugs. The neurological examination revealed dysarthria, dysmetria in both legs, ataxic gait and inability to walk without assistance due to several cerebellar ataxia affecting all limbs and trunk. His scale for the assessment and rating of ataxia score, in which $\leq 8$ points indicates the ability to walk unassisted, was 24.5. His muscle strength and sensory examinations were normal, and his deep tendon reflexes were normoactive. He did not have nystagmus or cognitive impairment. Laboratory evaluation revealed high serum anti-Ro/SSA and anti-La/SSB antibody levels $(\geq 1,200$ and $198 \mathrm{U} / \mathrm{mL}$, respectively) and antinuclear antibody (ANA) positivity $(1: 80)$. He was negative for anti-dsDNA, anti-Sm and anti-phospholipid (cardiolipin and $\beta 2$ glycoprotein) antibodies. Paraneoplastic (anti-Hu, nti-Ri, anti-Yo, anti-Tr, antiPNMA2 and anti-CV2), anti-GAD and anti-thyroglobulin antibodies were not detected. Both Schirmer's and fluorescein tests were positive, indicating that the patient also had dry eye. A salivary gland biopsy was then performed, revealing a lymphocytic infiltration around the salivary gland duct (Fig. 1a, b). The CSF showed increased anti-Ro/SSA antibodies at 15.9 $\mathrm{U} / \mathrm{mL}$ (normal range: $<7.0 \mathrm{U} / \mathrm{mL}$ ) and negative anti-La/SSB antibodies $(<1.0 \mathrm{U} / \mathrm{mL})$. This patient was then diagnosed with $\mathrm{SjS}$ according to the American-European Consensus Group's criteria [5]. Intravenous methylprednisolone (1 g/day) was then administered for 3 days, and maintenance therapy with oral methylprednisolone was continued for 4 months. In addition, the patient received intravenous immunoglobulin treatments twice during his hospitalization. Four months later, the patient had significant clinical improvement, with a scale for the assessment and rating of ataxia score of 12. After 1 year, brain magnetic resonance imaging (MRI) showed cerebellar atrophy (Fig. 1c), compared with the MRI obtained at the time of the patient's hospital admission (Fig. 1d).

Patient 2 (SjS with chronic inflammatory demyelinating

\section{polyradiculoneuropathy (CIDP))}

A 53-year-old male patient was admitted to our neurology department with complaints of numbness and weakness in all limbs, which emerged 3 years earlier and gradually worsened. He had a history of hypertension and hyperlipidemia. His cranial nerves were intact, and there were no overt symptoms of cerebellar ataxia by neurological examination. Deep tendon reflexes were not present in the upper and lower extremities, and there were no pathological reflexes. Muscle atrophy and weakness (Medical Research Center muscle scale, grade: R4-/L4) were noted in the distal muscles of lower extremities. Sensory disturbance (dysesthesia and hypoesthesia) was observed along the dermatomes of the left chest, left abdomen, left back and both extremities. A slight decrease in position and vibration sense was observed in both feet. He showed a wadding gait and inability to walk with a tandem gait. The serum anti-Ro/SSA and anti-La/SSB antibody levels were 26.5 and $24.5 \mathrm{U} / \mathrm{mL}$, respectively. The levels of ANA, anti-dsDNA, anti-GM1 and anti-GQ1b were also within the normal limits. He was also negative for serum anti-neutrophil cytoplasmic antibodies (ANCAs) targeting myeloperoxidase (MPO) and proteinase 3 (PR3). A lip biopsy showed lymphocyte infiltration around the salivary gland duct. A series of electromyographic examinations showed polyradiculoneuropathy with demyelination, consistent with the electrodiagnostic criteria of CIDP. CSF specimens showed increased total protein levels $(96 \mathrm{mg} / \mathrm{dL})$, and both anti-Ro/SSA and anti-La/SSB antibodies were determined to be absent $(<1.0 \mathrm{U} / \mathrm{mL})$. Brain MRI did not reveal abnormalities, and the patient was diagnosed with $\mathrm{SjS}$ with CIDP.

We present patient 2 to show the differences between patients with $\mathrm{SjS}$ with peripheral nervous system (PNS) involvement and those with CNS involvement (patient 1). Anti-Ro/ SSA antibodies were positive in the serum, but negative in the CSF, in the patient with SjS who had PNS involvement without CNS involvement.

\section{Literature review}

We conducted a literature review by searching PubMed for articles from 1990 to 2019 using the keywords "Sjogren's syndrome", "cerebellar" or "cerebellum" and "Purkinje". Several patients with SjS who also developed cerebellar degeneration were previously reported to undergo autopsy, including the examination of cerebellum. We reprinted and modified the photomicrographs of cerebellar tissue sections from a patient whose neuropathological findings in cerebellum were reported by Nanri et al, with permission from the publisher ([6], figure 4, Creative Commons CC BY). We compared the cerebellar tissue section of that patient with a healthy human cerebellar tissue section.

\section{Immunohistochemistry}

This experimental study used healthy 8 -week-old C57BL/6N 

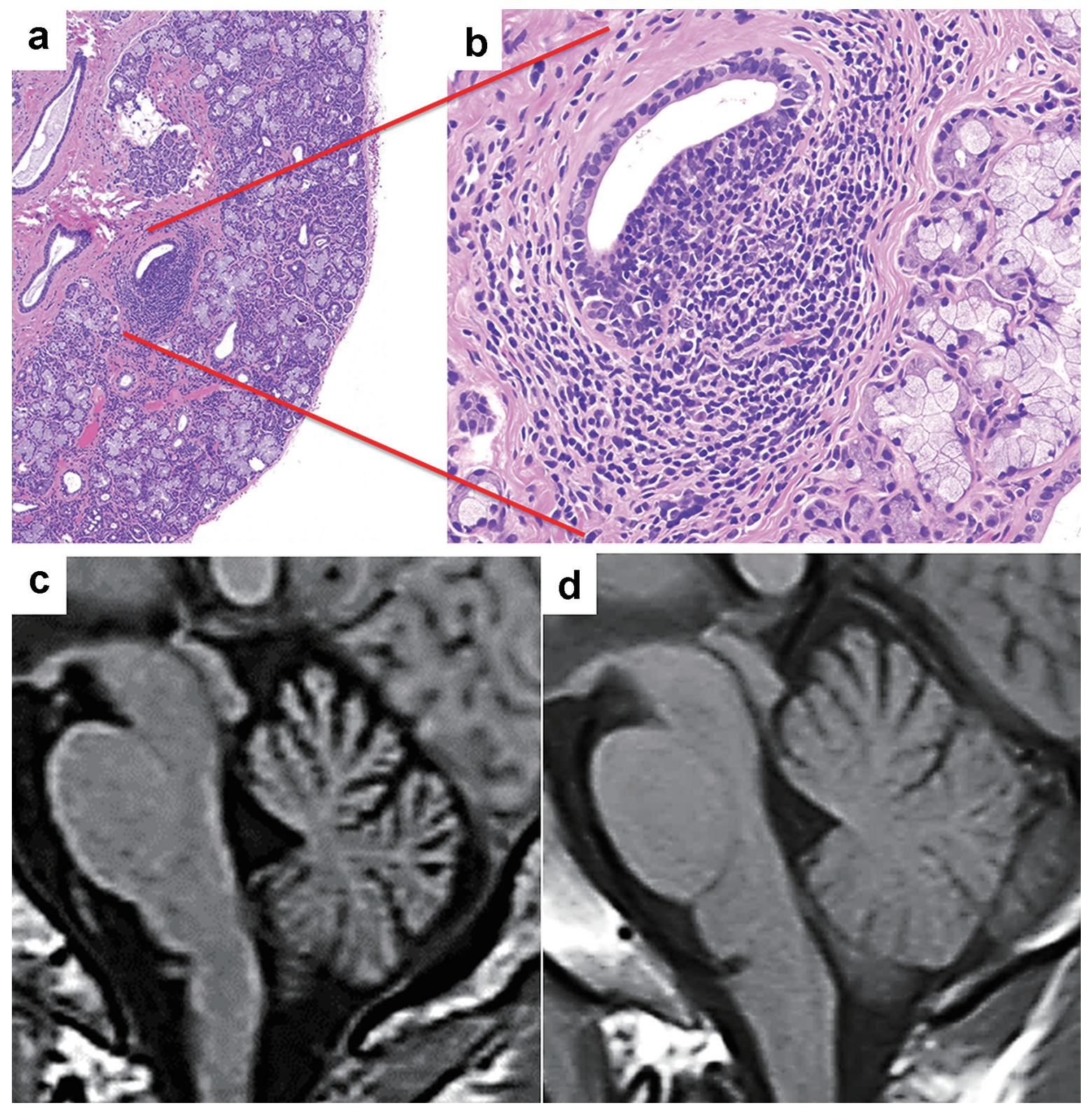

Figure 1. Patient 1 (Sjogren's syndrome with cerebellar degeneration). (a, b) Photomicrographs of a salivary gland tissue section showing mild lymphocytic infiltration between the acini and glands (hematoxylin and eosin stain, magnification for a: $\times 40$, b: $\times$ 200). (c, d) Sagittal views of the brain using magnetic resonance imaging T1 sequences. (c) The MRI performed 1 year after the initial presentation revealed cerebellar atrophy. (d) The initial MRI was unremarkable.

male mice. Animal experiments were approved by the Institutional Animal Experiment Committee of the Genostaff Co., Ltd, Tokyo, Japan, and complied with the Institutional Regulations for Animal Experiments and Fundamental Guidelines for the Proper Conduct of Animal Experiments and Related Activities in Academic Research Institutions under the Ministry of Education, Culture, Sports, Science, and Technology. Using immunohistochemistry, paraffin-embedded murine sagittal sections of the brain tissue were de-paraffined with xylene and rehydrated using graded ethanol and phosphatebuffered saline (PBS). Antigen retrieval was performed in a citrate buffer at pH 6 and given microwave treatment. Endog- enous peroxidase was blocked with $0.3 \% \mathrm{H}_{2} \mathrm{O}_{2}$ in methanol for $30 \mathrm{~min}$, followed by incubation with G-Block (Genostaff) and an avidin/biotin blocking kit (Vector). Sections were incubated with an anti-Ro52/TRIM21 rabbit polyclonal antibody (Affinity Biosciences) at $4{ }^{\circ} \mathrm{C}$ overnight; these were further incubated with biotin-conjugated anti-rabbit Ig (Dako) for $30 \mathrm{~min}$ at room temperature, followed by the addition of peroxidase-conjugated streptavidin (Nichirei) for $5 \mathrm{~min}$. Peroxidase activity was visualized using diaminobenzidine staining. The sections were counterstained with Mayer's hematoxylin (MUTO), were dehydrated and then were mounted with malinol (MUTO). 


\section{Results \\ Characteristics of cerebellar degeneration in patients with SjS}

We found 14 patients with previously reported $\mathrm{SjS}$, including our patient 1 , who also had developed cerebellar degeneration [6-18]. The characteristics of cerebellar degeneration in patients with $\mathrm{SjS}$ are provided in Table 1. The average age of onset was $48.9 \pm 19.4$ years. The male/female ratio was overwhelmingly female, with three men and 11 women. Cerebellar atrophy was observed with brain MRI in the majority of the patients as the disease progressed (12/14 cases). Three main symptoms suggested cerebellar disturbances: ataxia (14/14 cases), nystagmus (6/14 cases) and dysarthria (10/14 cases). A few patients had been diagnosed with $\mathrm{SjS}$ (2/14 cases), and many patients presented with cerebellar degeneration as the first manifestation of $\mathrm{SjS}$, as did our patient 1 .

In many case reports, steroid or immunosuppressive therapies were provided, and, despite the relatively good therapeutic responses to this type of therapy, sequelae remained in 11 of the 14 patients, including our patient 1 . In the majority of patients, cerebellar atrophy was observed after the onset of neurological symptoms (12/14 cases). Interestingly, Nanri et al reported on their SjS patient with cerebellar degeneration found on autopsy [6]. This patient had a selective loss of Purkinje cells, had no apparent degenerative changes in the efferent pathways, such as the dentate or vestibular nuclei and had no prominent inflammatory reaction (Fig. 2).

The most notable finding in the literature review was the positive rate of autoantibody detection (Table 1). Although anti-Ro/SSA antibodies in the serum were positive in all patients, anti-La/SSB antibodies in the serum were negative in five of the 14 patients $[7,12,16-18]$. In addition, our patient 2, who had SjS with PNS involvement but no CNS involvement, was negative for both anti-Ro/SSA and anti-La/SSB antibodies in the CSF. These findings suggest that anti-Ro/SSA antibodies, but not anti-La/SSB antibodies, are involved in the pathology of SjS-related cerebellar degeneration. Therefore, first, we have studied in mouse brain tissue and examined whether the Ro/SSA (Ro52/TRIM21) protein was expressed in the cerebellum of mice using immunohistochemistry.

\section{Ro52/TRIM21 expression in the murine brain}

We demonstrated that Ro52/TRIM21 expression was easily detected throughout the brain, including the hippocampus, cerebral cortex and cerebellum (Fig. 3a) and that the control antibody did not exhibit any specific staining patterns (Fig. 3b). Ro52/TRIM21 expression was clearly detected in the murine cerebellum using anti-Ro52/TRIM21 antibodies (Fig. 4a) as compared with the negative staining using normal rabbit Ig (Fig. 4b). In particular, high Ro52/TRIM21 expression was observed in the cytoplasm of Purkinje cells (Fig. 4a), suggesting that Ro52/TRIM21-expressing cells in the cerebellum are major targets for anti-Ro52/TRIM21. In addition, Ro52/TRIM21

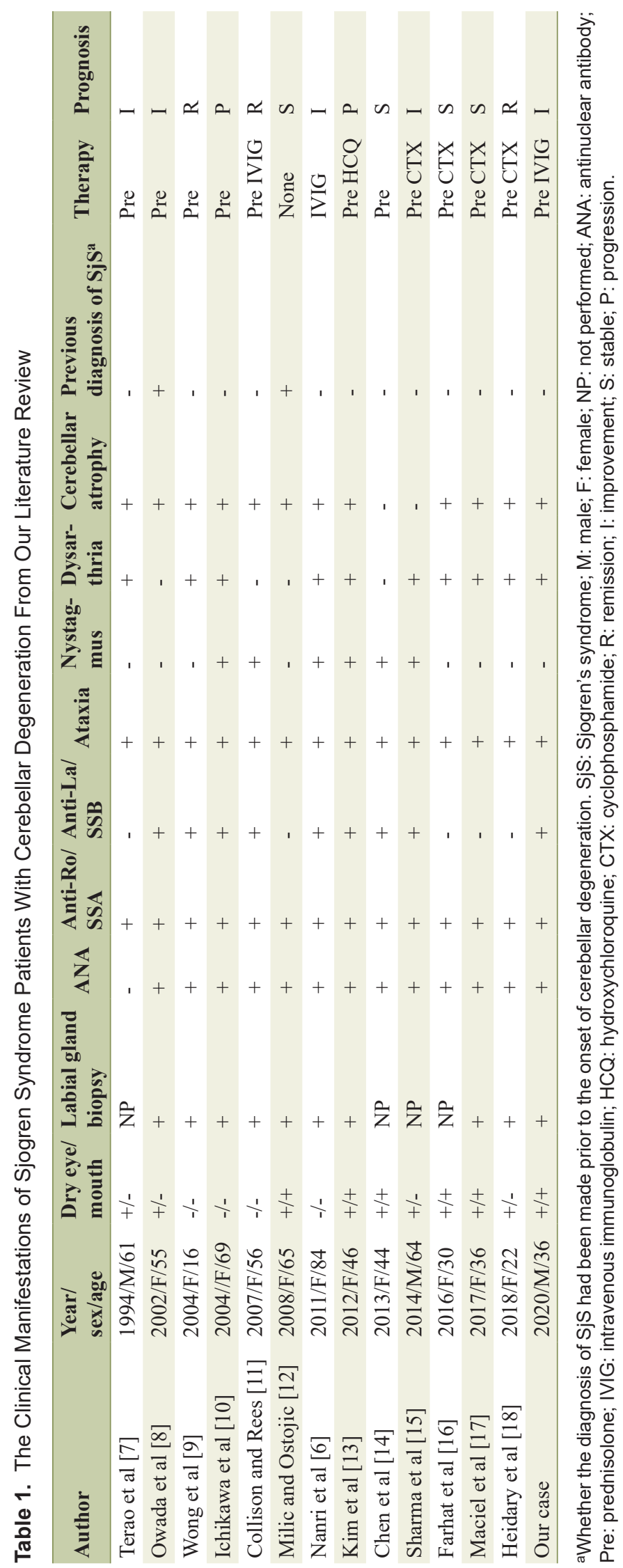




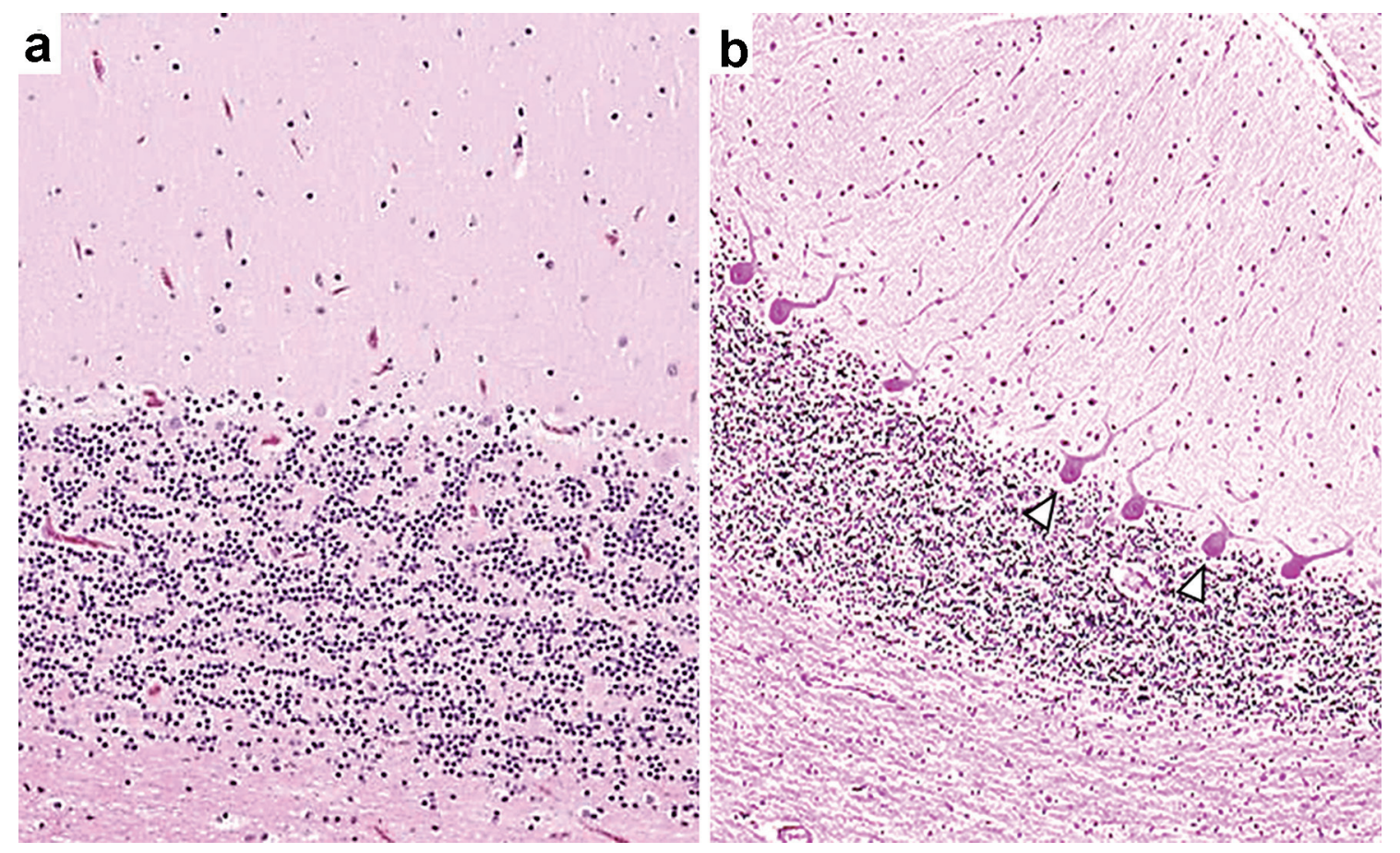

Figure 2. A selective loss of Purkinje cells in a patient with Sjogren's syndrome with cerebellar degeneration. The patient was an 84-year-old woman who presented with nystagmus, dysarthria and ataxia in all limbs. Anti-Ro/SSA antibodies concentrations were determined to be $>500 \mathrm{U} / \mathrm{mL}$, and anti-La/SSB antibodies were $41.1 \mathrm{U} / \mathrm{mL}$. Intravenous immunoglobulin therapy was moderately effective. The patient died of pneumonia at age 85 years; an autopsy was then performed [6]. (a) A photomicrograph shows a mild-to-moderate decrease in the number of Purkinje cells in association with mild Bergman gliosis elsewhere in the cerebellar cortex. (b) This photomicrograph shows a normal human cerebellar tissue section with an appropriate number of Purkinje cells. Hematoxylin and eosin stain. The photomicrographs of tissue sections from this patient were reprinted and modified with permission from the publisher ([6], figure 4, Creative Commons CC BY). Arrowheads indicate Purkinje cells.

expression also was detected in the cytoplasm of the neurons on the cortex (Fig. 5a) and the hippocampus (Fig. 5b), and high levels of staining were observed using anti-Ro52/TRIM21 antibodies in the cytoplasm of the neurons in the CA3 region of the hippocampus on high magnification image (Fig. 5c).

\section{Discussion}

The most notable findings in this study were as follows: 1) Although anti-Ro/SSA antibodies were found in all patients, there were patients in whom anti-La/SSB antibodies were negative; 2) Anti-Ro/SSA antibodies were observed in both the serum and CSF; 3) In the SjS patient who had PNS involvement without CNS involvement (our patient 2), anti-Ro/SSA antibodies were negative in the CSF; 4) Cerebellar atrophy was observed after the onset of cerebellar degeneration in patients with $\mathrm{SjS}$, and sequelae have remained in most cases; 5) A selective loss of Purkinje cells was found in the histological sections of the brain in one patient with cerebellar degeneration; 6) Ro52/TRIM21 expression was detected throughout the murine brain, including the hippocampus, cerebral cortex and cerebellum; and 7) High Ro52/TRIM21 expression was observed in Purkinje cells.

In the literature review, we were able to determine that all patients had anti-Ro/SSA antibodies, but some of those were negative for anti-La/SSB antibodies. This finding was counter to studies showing that anti-La/SSB antibodies are usually present with anti-Ro/SSA antibodies in serum, while anti-Ro/ SSA antibodies could be detected without the presence of anti$\mathrm{La} / \mathrm{SSB}$ antibodies, suggesting the latter antibodies are not associated with the pathophysiological mechanism of cerebellar degeneration in $\mathrm{SjS}$.

Furthermore, anti-Ro/SSA antibodies were observed in both the serum and CSF of our patient 1 but were negative in the CSF of our patient 2 who did not have CNS involvement. It is essential to detect antineuronal autoantibodies in the CSF of patients with autoimmune diseases of the CNS. The presence of anti-Ro/SSA antibodies in the CSF suggests that there is a disruption of the blood-brain barrier, that autoantibody-mediated neuroinflammation is involved in the pathophysiological mechanisms of CNS disease, and that an immune-mediated neurological disease is likely [19].

Anti-Ro/SSA antibodies are typically described as being associated with $\mathrm{SjS}$; they have been determined to have differential actions by two target proteins, Ro52/TRIM21 (52 kDa) and Ro60 (60 kDa), that are biochemically and immunologically distinct [20]. The seropositivity prevalence rates were approximately $70 \%$ for anti-Ro52/TRIM 21 antibodies, $40 \%$ for anti-Ro60 antibodies and 50\% for anti-La/SSB antibodies; in addition, $63.2 \%$ of the patients with anti-Ro52/TRIM21 antibodies in serum also had anti-Ro60 antibodies [3, 21]. 


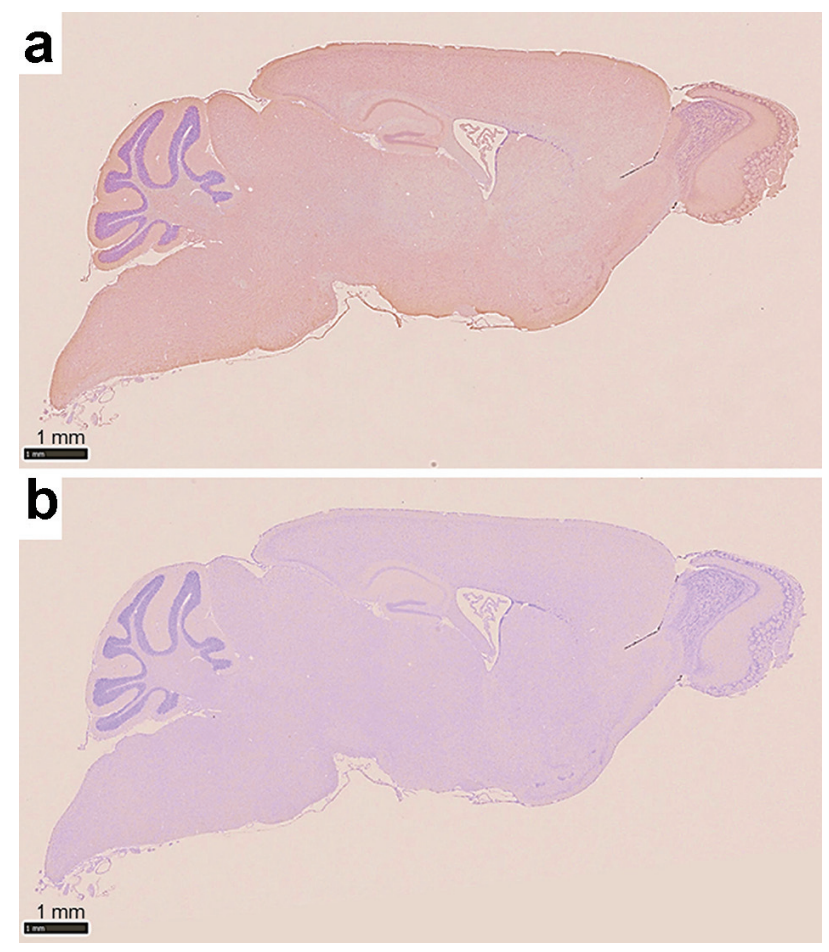

Figure 3. Photomicrographs of immunohistochemistry of a sagittal section of the murine brain. (a) The section is stained with a commercially available anti-Ro52/TRIM21 antibody showing Ro52/TRIM21 expression throughout the entire brain, including the hippocampus, cerebral cortex and cerebellum. (b) Negative control using an unrelated control antibody (anti-rabbit Ig) showing a lack of background staining (counterstain: hematoxylin).

Clinical findings have been shown to be different between the patients with anti-Ro52/TRIM21 and anti-Ro60 antibodies [20]. The Ro52/TRIM21 gene is $8.8 \mathrm{~kb}$ in size, located on chromosome 11 , and is a cytoplasmic protein that belongs to the TRIM family. This protein has been identified to be involved in protein ubiquitination, proinflammatory states (interleukin 2) and apoptotic mechanisms; is suggested to play an important role in the regulation of inflammatory responses; and is upregulated at sites of autoimmune inflammation [22]. According to one study, intrathecal production of anti-Ro52/ TRIM21 antibodies was observed in patients with $\mathrm{SjS}$ with CNS involvement [4]. In addition, Ro52/TRIM21 was recognized by anti-Hu antibodies, which are onconeural antibodies for paraneoplastic neurological syndromes, including paraneoplastic cerebellar degeneration [23].

Although these results suggest that the Ro52/TRIM2 protein is involved to a greater extent in SjS-related cerebellar degeneration compared with the Ro60 protein, to the best of our knowledge, the distribution of this protein in the human brain remains to be unknown. In this study, we demonstrated that Ro52/TRIM21 was expressed throughout the murine brain, including the hippocampus, cerebral cortex and cerebellum. Moreover, because the histopathological hallmark of autoimmune cerebellar degeneration is a severe loss of Purkinje cells [24], we might be able to confirm this point from the results of our immunohistochemistry analysis and previous autopsy case

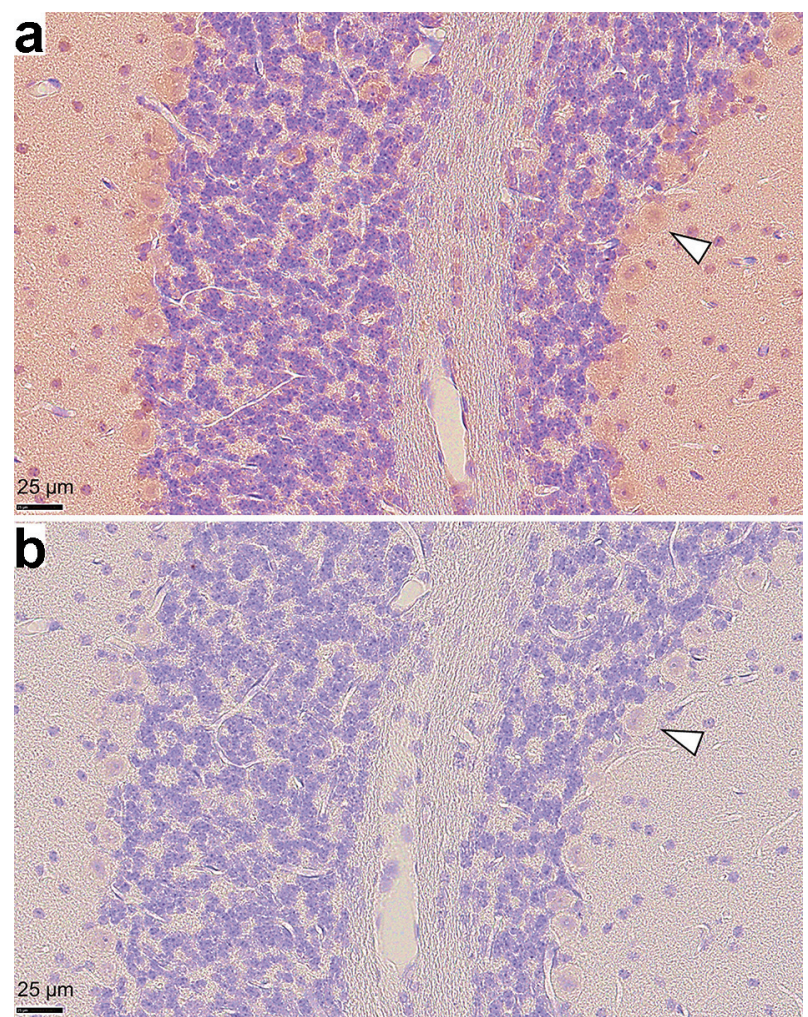

Figure 4. Photomicrographs of immunohistochemistry on murine cerebellar tissue sections. (a) Ro52/TRIM21 expression is shown using an anti-Ro52/TRIM21 antibody, but (b) is not present after staining with an anti-rabbit Ig (negative control). High Ro52/TRIM21 expression was observed in the cytoplasm of Purkinje cells. Arrowheads indicate representative Purkinje cells (magnification: $\times 40$, counterstain: hematoxylin)

report [6]. Although the pathogenic mechanisms of autoantibodies against Ro/SSA in autoimmune disease has remained unclear, the hypothesis exists that anti-Ro/SSA antibodies might cause direct damage to cells and that anti-Yo antibodies, which are onconeural antibodies, are associated with paraneoplastic cerebellar degeneration [25, 26].

Some studies have reported that anti-Ro52/TRIM21 autoantibodies can penetrate the cytoplasm and inhibit the function of the Ro52/TRIM21 protein, which negatively regulates proinflammatory cytokines and degrades Ro52/TRIM21 E3 ubiquitin ligase activity $[27,28]$. Furthermore, as anti-Ro/SSA antibodies were identified in the CSF of the patients with $\mathrm{SjS}$ with cerebellar degeneration, it is possible that these antibodies might attack Purkinje cells, reducing Purkinje cell functions and activities, and can cause symptoms of neurological degeneration, resulting in cerebellar atrophy. Several reports have shown the presence of serum anti-Ro/SSA antibodies in patients with $\mathrm{SjS}$ with limbic encephalitis [11, 29]. We demonstrated that Ro52/TRIM21 was expressed in the neurons of the cortex and hippocampus, which might indicate that anti-Ro/SSA antibodies are involved in other CNS diseases in addition to cerebellar degeneration.

The limitations of this study include the need for proof that anti-La/SSB antibodies are completely unrelated with $\mathrm{SjS}$ with cerebellar degeneration. The number of cases is so 


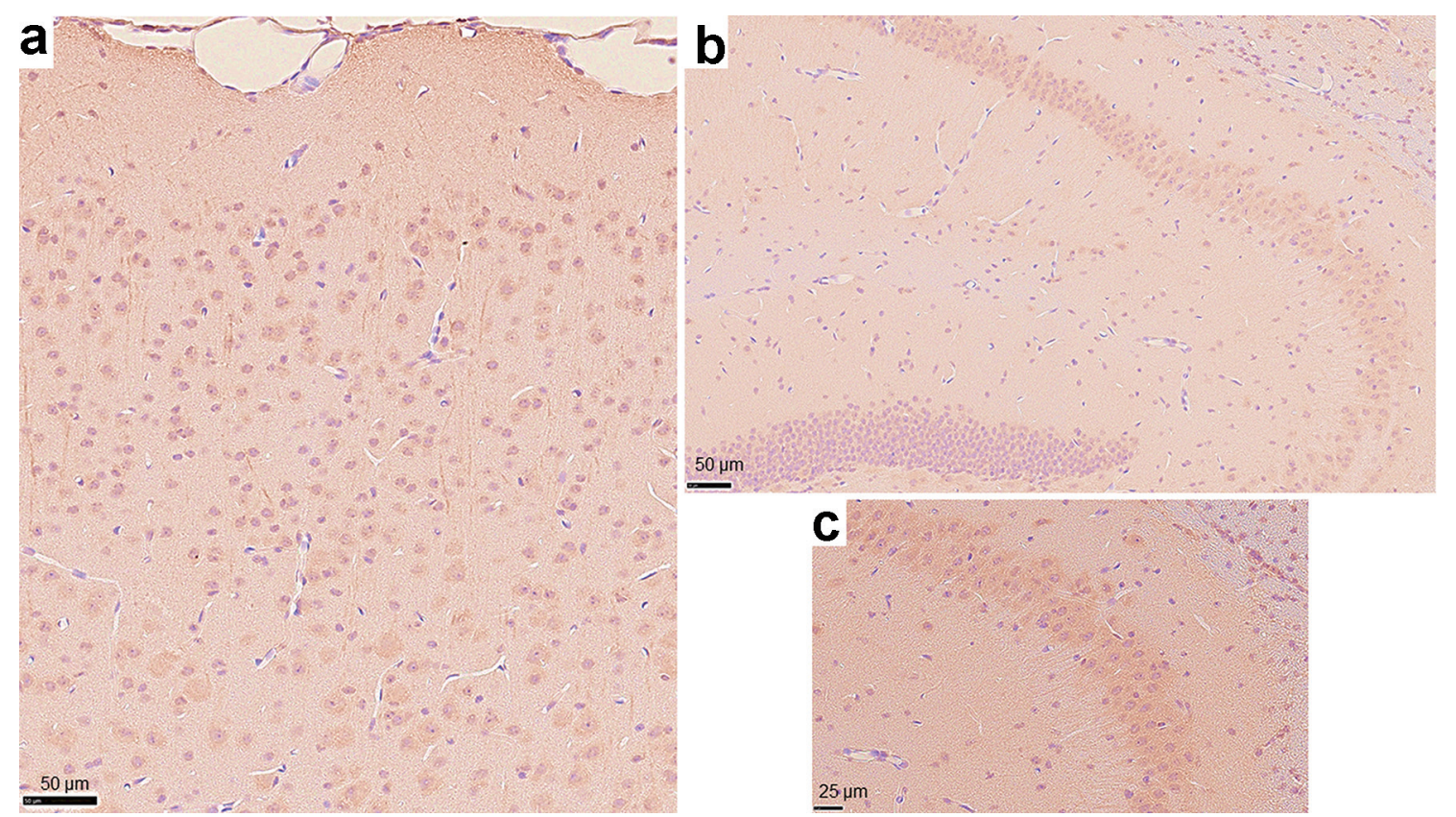

Figure 5. Photomicrographs of immunohistochemistry on other regions of the murine brain using the anti-Ro52/TRIM21 antibody. Cells that stained positively with the anti-Ro52/TRIM21 antibody were observed throughout (a) the cerebral cortex and (b) the hippocampus (magnification: $\times 20$ ). (c) High magnification image representing the CA3 region of the hippocampus (magnification: $\times 40$, counterstain: hematoxylin).

small that it is difficult to present the difference in positive rates between anti-Ro/SSA and anti-La/SSB as statistically significant. However, the observation that anti-Ro/SSA antibodies are positive in all patients with cerebellar degeneration while anti-La/SSB are negative in a minority is thus especially important. The strengths of this study include experimental results that high Ro/SSA expression was observed in Purkinje cells, which supports the hypothesis that Ro/SSA is involved in the pathology of cerebellar degeneration with $\mathrm{SjS}$. Further studies are required to clarify the relevance of $\mathrm{SjS}$ to cerebellar degeneration and the pathogenic mechanisms of the antineuronal antibodies against Ro/SSA to determine how they may cause CNS dysfunction.

\section{Conclusion}

In this literature review and the analysis of our patients in the case studies, we evaluated the characteristics of cerebellar degeneration associated with $\mathrm{SjS}$. We found high Ro52/TRIM21 expression in the Purkinje cells in the histological sections of the cerebellum. Thus, we can conclude that anti-Ro/SSA antibodies are the antineuronal antibodies involved in the cerebellar degeneration of patients with $\mathrm{SjS}$ and that the finding of anti-Ro/SSA antibodies in CSF might serve as a useful biomarker for SjS-related CNS diseases in the clinical setting. Additional studies are warranted to confirm these observations and to clarify the molecular and pathological mechanisms of Ro/SSA antibodies as antineuronal antibodies. Finally, determining how these autoantibodies cause CNS dysfunction is also necessary.

\section{Acknowledgments}

The authors would like to thank the Genostaff Co., Ltd for technical support of immunohistochemistry.

\section{Financial Disclosure}

This research did not receive any specific grant from funding agencies in the public, commercial or not-for-profit sectors.

\section{Conflict of Interest}

The authors declare no conflict of interest.

\section{Informed Consent}

Written informed consent for publication was obtained and is available upon request.

\section{Author Contributions}

ST, TS, RH and HK developed the main conceptual idea and research design. ST and TO performed experiment and data acquisition. ST, RH and HK analyzed the data. ST wrote the manuscript. ST, TO and HK edited manuscript. All authors approved the final version of the manuscript. 


\section{Data Availability}

The authors declare that data supporting the findings of this study are available within the article. The datasets are available from the corresponding author upon reasonable request.

\section{References}

1. Fox RI. Sjogren's syndrome. Lancet. 2005;366(9482):321331.

2. Carvajal Alegria G, Guellec D, Mariette X, Gottenberg JE, Dernis E, Dubost JJ, Trouvin AP, et al. Epidemiology of neurological manifestations in Sjogren's syndrome: data from the French ASSESS Cohort. RMD Open. 2016;2(1):e000179.

3. Jonsson R, Brokstad KA, Jonsson MV, Delaleu N, Skarstein K. Current concepts on Sjogren's syndrome classification criteria and biomarkers. Eur J Oral Sci. 2018;126(Suppl 1):37-48.

4. Megevand P, Chizzolini C, Chofflon M, Roux-Lombard P, Lalive PH, Picard F. Cerebrospinal fluid anti-SSA autoantibodies in primary Sjogren's syndrome with central nervous system involvement. Eur Neurol. 2007;57(3):166-171.

5. Vitali C, Bombardieri S, Jonsson R, Moutsopoulos HM, Alexander EL, Carsons SE, Daniels TE, et al. Classification criteria for Sjogren's syndrome: a revised version of the European criteria proposed by the American-European Consensus Group. Ann Rheum Dis. 2002;61(6):554558.

6. Nanri K, Shibuya M, Taguchi T, Hasegawa A, Tanaka N. Selective loss of Purkinje cells in a patient with antigliadin-antibody-positive autoimmune cerebellar ataxia. Diagn Pathol. 2011;6:14.

7. Terao Y, Sakai K, Kato S, Tanabe H, Ishida K, Tsukamoto T. Antineuronal antibody in Sjogren's syndrome masquerading as paraneoplastic cerebellar degeneration. Lancet. 1994;343(8900): 790 .

8. Owada K, Uchihara T, Ishida K, Mizusawa H, Watabiki $\mathrm{S}$, Tsuchiya $\mathrm{K}$. Motor weakness and cerebellar ataxia in Sjogren syndrome-identification of antineuronal antibody: a case report. J Neurol Sci. 2002;197(1-2):79-84.

9. Wong S, Pollock AN, Burnham JM, Sherry DD, Dlugos DJ. Acute cerebellar ataxia due to Sjogren syndrome. Neurology. 2004;62(12):2332-2333.

10. Ichikawa H, Ishihara K, Fujimoto R, Katoh T, Arai M, Kawamura M, Nakano I. An autopsied case of Sjogren's syndrome with massive necrotic and demyelinating lesions of the cerebellar white matter. J Neurol Sci. 2004;225(1-2):143-148.

11. Collison K, Rees J. Asymmetric cerebellar ataxia and limbic encephalitis as a presenting feature of primary Sjogren's syndrome. J Neurol. 2007;254(11):1609-1611.

12. Milic V, Ostojic P. Cerebellar ataxia in a patient with primary Sjogren's syndrome after treatment with chloroquine. Rheumatol Int. 2008;28(12):1295-1296.

13. Kim MJ, Lee MC, Lee JH, Chung SJ. Cerebellar degeneration associated with Sjogren's syndrome. J Clin Neurol.
2012;8(2):155-159.

14. Chen YW, Lee KC, Chang IW, Chang CS, Hsu SP, Kuo HC. Sjogren's syndrome with acute cerebellar ataxia and massive lymphadenopathy: a case report. Acta Neurol Taiwan. 2013;22(2):81-86.

15. Sharma R, Chilukuri V, Sarma AK, Gokhale S. Primary Sjogren's syndrome presenting as acute cerebellitis. J Clin Neurosci. 2014;21(3):508-509.

16. Farhat E, Zouari M, Abdelaziz IB, Drissi C, Beyrouti R, Hammouda MB, Hentati F. Progressive cerebellar degeneration revealing Primary Sjogren Syndrome: a case report. Cerebellum Ataxias. 2016;3:18.

17. Maciel R, Camargos S, Cardoso F. Subacute cerebellar degeneration as the first manifestation of Sjogren's syndrome. Mov Disord Clin Pract. 2017;4(4):637-638.

18. Heidary M, Alesaeidi S, Afshari K. Cerebellar degeneration in primary Sjgren syndrome. BMJ Case Rep. 2018;2018.

19. Shimizu F, Nishihara H, Kanda T. Blood-brain barrier dysfunction in immuno-mediated neurological diseases. Immunol Med. 2018;41(3):120-128.

20. Schulte-Pelkum J, Fritzler M, Mahler M. Latest update on the Ro/SS-A autoantibody system. Autoimmun Rev. 2009;8(7):632-637.

21. Garberg H, Jonsson R, Brokstad KA. The serological pattern of autoantibodies to the Ro52, Ro60, and La48 autoantigens in primary Sjogren's syndrome patients and healthy controls. Scand J Rheumatol. 2005;34(1):49-55.

22. Oke V, Wahren-Herlenius M. The immunobiology of Ro52 (TRIM21) in autoimmunity: a critical review. J Autoimmun. 2012;39(1-2):77-82.

23. Manley G, Wong E, Dalmau J, Elkon K, Posner J, Furneaux H. Sera from some patients with antibody-associated paraneoplastic encephalomyelitis/sensory neuronopathy recognize the Ro-52K antigen. J Neurooncol. 1994;19(2):105-112.

24. Dalmau J, Rosenfeld MR. Paraneoplastic syndromes of the CNS. Lancet Neurol. 2008;7(4):327-340.

25. Yoshimi R, Ueda A, Ozato K, Ishigatsubo Y. Clinical and pathological roles of Ro/SSA autoantibody system. Clin Dev Immunol. 2012;2012:606195.

26. Greenlee JE, Clawson SA, Hill KE, Wood B, Clardy SL, Tsunoda I, Carlson NG. Anti-Yo antibody uptake and interaction with its intracellular target antigen causes Purkinje cell death in rat cerebellar slice cultures: a possible mechanism for paraneoplastic cerebellar degeneration in humans with gynecological or breast cancers. PLoS One. 2015;10(4): $\mathrm{e} 0123446$.

27. Mallery DL, McEwan WA, Bidgood SR, Towers GJ, Johnson CM, James LC. Antibodies mediate intracellular immunity through tripartite motif-containing 21 (TRIM21). Proc Natl Acad Sci U S A. 2010;107(46):19985-19990.

28. Espinosa A, Hennig J, Ambrosi A, Anandapadmanaban M, Abelius MS, Sheng Y, Nyberg F, et al. Anti-Ro52 autoantibodies from patients with Sjogren's syndrome inhibit the Ro52 E3 ligase activity by blocking the E3/E2 interface. J Biol Chem. 2011;286(42):36478-36491.

29. Coban A, Ozyurt S, Meric K, Misirli H, Tuzun E, Turkoglu R. Limbic encephalitis associated with Sjogren's syndrome: report of three cases. Intern Med. 2016;55(16):2285-2289. 